\title{
Interchain and intrachain emission branching in polymer light-emitting diode doped by organic molecules
}

\author{
D. Krautz, ${ }^{1}$ E. Lunedei, ${ }^{2}$ J. Puigdollers ${ }^{3}$ G. Badenes, ${ }^{1}$ R. Alcubilla, ${ }^{3}$ and S. Cheylan ${ }^{1, a)}$ \\ ${ }^{1}$ ICFO-Institut de Ciencies Fotoniques, Mediterranean Technology Park, \\ 08860 Castelldefels (Barcelona), Spain \\ ${ }^{2}$ ISMN-CNR, Institute for the Study of Nanostructured Materials, 40129 Bologna, Italy \\ ${ }^{3}$ Departamento de Ingeniería Electrónica, Universidad Politécnica de Cataluña-UPC, \\ 08034 Barcelona, Spain
}

(Received 15 May 2009; accepted 27 November 2009; published online 20 January 2010)

\begin{abstract}
A blend of the polymer poly[2-(2-ethylhexyloxy)-5-methoxy-1,4-phenylenevinylene] (MEH-PPV) and the electron-transport molecule tris-(8-hydroxyquinoline) aluminum ( $\left.\mathrm{Alq}_{3}\right)$ has been investigated by means of electroluminescence and fluorescence spectroscopy, upon variation of the $\mathrm{Alq}_{3}$ content in the blend. A decreased interchain emission is observed upon increasing $\mathrm{Alq}_{3}$ content, due to lower packing of the MEH-PPV chains which leads to a reduction in the interchain interaction, excimer formation, and emission probability. A branching of MEH-PPV interchain and intrachain emissive contributions is clearly time resolved and analyzed as a function of the $\mathrm{Alq}_{3}$ content. At high doping concentration, direct emission from $\mathrm{Alq}_{3}$ molecules is observed. (C) 2010 American Institute of Physics. [doi:10.1063/1.3276271]
\end{abstract}

Conjugated polymers have been extensively investigated due to their interesting physical properties as well as for their large potential for electroluminescent applications. Recently, polymer blends represent an alternative approach to new materials with improved performance for use in semiconductor devices. Numerous reports have demonstrated that utilizing the blending techniques, single-layer polymer light-emitting device (PLED) with high performance can be achieved. ${ }^{1-5}$ Both the carrier injection and transport can be improved by carefully selecting the materials and their proportions in the blend. ${ }^{1-5}$ Furthermore, the dopant introduction produces a dilution effect of the polymer solution and reduces polymer interchain interaction, leading to sharper EL spectra with higher color purity than those of the neat polymer film, depending on the dopant concentration. ${ }^{5-7}$

In conjugated polymers, intrachain singlet excitons can be generated by photons absorption or via carrier-injection followed by electron-hole pair formation on a single chain. These excitations are usually extended over some ( $\sim 6$ or 7 ) repeating units. ${ }^{8}$ The diffusion of the excitation along the polymer chain as well as between different chains is also possible. Two "molecules" (i.e., conjugated lengths), one neutral and one in the excited state, belonging to two different sites of the same chain or to different polymer chains can interact resonantly forming one interchain excitation as an excimer. Excimers exhibit by a long radiative lifetime because the transition from the excimer state to the ground state is usually forbidden by symmetry. ${ }^{9}$ They exhibit redshifted, broad emission spectra. Nevertheless, since interchain excitations decay pathway is mainly nonradiative, they act as effective luminescence quencher of singlet intrachain states.

In this work, we investigate the branching of intrachain and interchain contribution to the emission of the polymer (MEH-PPV), doped by the electron transporter $\mathrm{Alq}_{3}$. Progressively passing from low to high concentrations of $\mathrm{Alq}_{3}$

\footnotetext{
a) Author to whom correspondence should be addressed. Electronic mail: stephanie.cheylan@icfo.es.
}

in the blend, it is possible to alter the formation of MEHPPV excimers (interchain species) due to the segregation of the polymer chains by the $\mathrm{Alq}_{3}$ molecules and in this way, favor the intrachain (polymer exciton) emission. Timeresolved spectroscopy evidences the dynamic of excitation transfer from intrachain to interchain species in MEH-PPV and from $\mathrm{Alq}_{3}$ to MEH-PPV for varying $\mathrm{Alq}_{3}$ content.

$\mathrm{MEH}-\mathrm{PPV}$ and $\mathrm{Alq}_{3}$ were dissolved in chloroform separately and then mixed at different ratios. The devices structure was ITO/PEDOT $(25 \mathrm{~nm}) / \mathrm{Blend}(155-230 \mathrm{~nm}) / \mathrm{Al}$. Current density $(J / V)$ and luminance $(L / V)$ versus voltage curves were measured in darkness and inert atmosphere by a Keithley 236 Source Measure Units and a calibrated photodiode. EL spectra were collected by an Ocean Optics USB4000 spectrometer. Steady-state photoluminescence (PL) measurements were performed, exciting with the 370 $390 \mathrm{~nm}$ spectra region of a halogen white light. A pulsed laser diode at $405 \mathrm{~nm}$ (Picoquant), with $70 \mathrm{ps}$ pulse width, at a repetition rate of $1 \mathrm{MHz}$ was used for the time-resolved PL (TRPL). The sample fluorescence was fed into a $0.3 \mathrm{~m}$ focal length monochromator (Acton SpectraPro-2300i) and detected with a cooled Hamamatsu photomultiplier tube (H7422-20). A Picoquant TimeHarp 100 board (inverted start-stop mode) measured the time correlated single photon counting.

The devices electrical characterizations show that for low $\mathrm{Alq}_{3}$ content $(<35 \%)$, the $J / V$ curves are similar to the pure MEH-PPV device, but an increased luminance is observed (Fig. 1). For larger amount of $\mathrm{Alq}_{3}(>35 \%)$, the current density constantly decreases upon increasing $\mathrm{Alq}_{3}$ amount and the measured luminance is comparable or lower than that of the reference device. Such behavior has been observed in devices based on blends of electron transport materials and MEH-PPV. ${ }^{3-5}$ The electron transport material favors the injection and transport of electrons throughout the blend layer. The luminance shows a fast increase with increasing $\mathrm{Alq}_{3}$ content, reaching a maximum for $\mathrm{Alq}_{3}$ contents between $9 \%$ and $15 \%$. As more electrons are injected and enter the active layer, they easily recombine with holes 


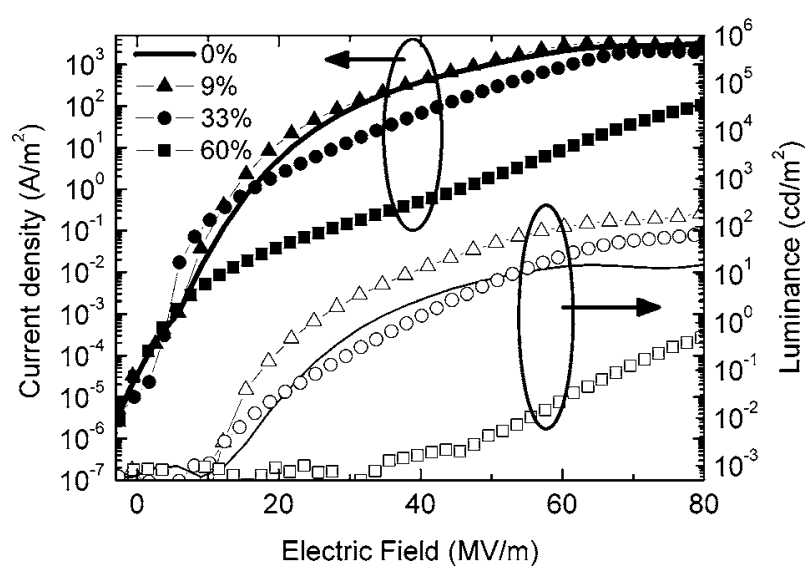

FIG. 1. Current density (filled symbols) and luminance (open symbols) vs electric field curves of the blend-based device for various percentages of $\mathrm{Alq}_{3}$ in the blend.

present in the layer, resulting in a drastic increase in luminance. Since small amount of $\mathrm{Alq}_{3}$ in the blend do not provide efficient hole blocking, the current flow is still high. For higher $\mathrm{Alq}_{3}$ concentration, the overall current density decreases continuously upon increasing $\mathrm{Alq}_{3}$ content as the confinement of the holes becomes more efficient due to a denser $\mathrm{Alq}_{3}$ network. As the separation of the polymer chains by $\mathrm{Alq}_{3}$ insertion becomes larger, the interchain polymer interaction decreases, such as the interchain charge transport, ${ }^{10}$ thus reducing the carrier mobility. The threshold voltage, constant around $4 \mathrm{~V}$, increases for $\mathrm{Alq}_{3}$ content above $33 \%$.

The reference device exhibits a broad EL band centered at $600 \mathrm{~nm}$ (Fig. 2) which is assigned to the convolution of the emission of intrachain excitons and interchain species. ${ }^{10,11}$ Such a broad structure is observed unchanged up to $33 \% \mathrm{Alq}_{3}$. Above $33 \%$, as the $\mathrm{Alq}_{3}$ amount increases, the low-energy component of this emission loses intensity (marked Ex on Fig. 2), leaving a broadband emission peaked at $570 \mathrm{~nm}$ with a shoulder at $610 \mathrm{~nm}$. The features at 570 and $610 \mathrm{~nm}$ are assigned to $(0 \rightarrow 0)$ and $(0 \rightarrow 1)$ fluorescence transitions of the polymer, respectively. ${ }^{12}$ The nodal point at $520 \mathrm{~nm}$ (indicated by a star) ensures that no apparent shift of the curves is present, but only a different balancing between the two luminescence components. As the polymer chains become separated by the electron transporting small molecules, the emission appears to be dominated by single chain

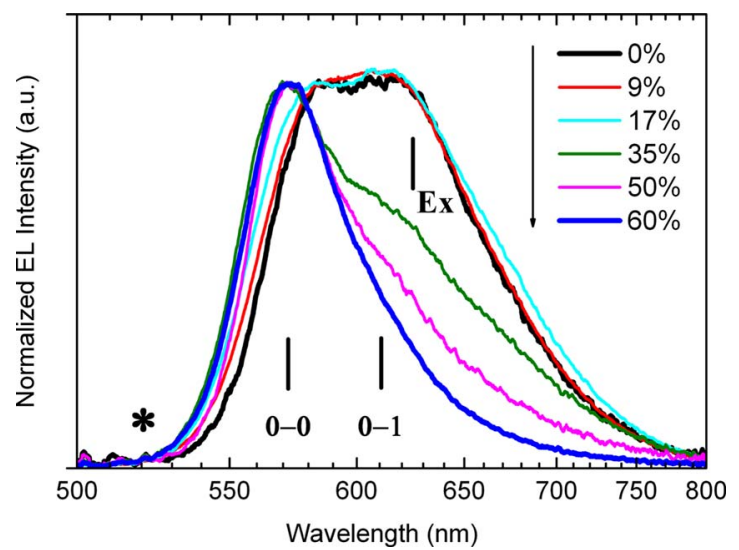

FIG. 2. (Color online) Normalized EL spectra for various $\mathrm{Alq}_{3}$ concentrations in the blend as active layer. The ${ }^{*}$ indicates a nodal point at $520 \mathrm{~nm}$, while the $(0 \rightarrow 0)$ and $(0 \rightarrow 1)$ indicate the fluorescence transitions.

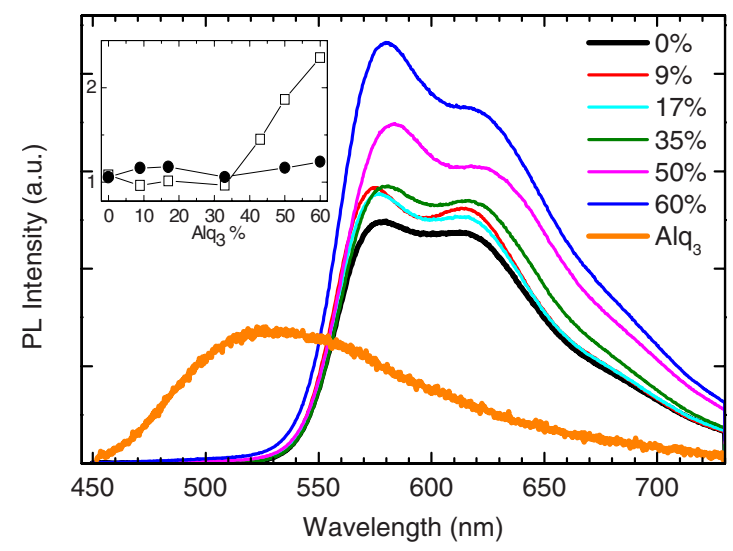

FIG. 3. (Color online) CW PL of $\mathrm{Alq}_{3}$ (solid orange curve), MEH-PPV (solid black curve) and the blend for $\mathrm{Alq}_{3}$ contents from 5\% up to $60 \%$. Inset shows the intensity ratio of the interchain and the intrachain emission for both EL (open square) and PL (filled circle) spectra.

MEH-PPV exciton. Furthermore, the EL spectra show no $\mathrm{Alq}_{3}$ contribution.

For all $\mathrm{Alq}_{3}$ concentrations, the blend fluorescence shows similar features to the pure MEH-PPV spectra (Fig. 3 ), with again no visible contribution from $\mathrm{Alq}_{3}$. The intensity ratio (inset Fig. 3) between the first emission band (intrachain) and the low energy emission (interchain) increases with $\mathrm{Alq}_{3}$ concentration, as a result of the reduction in excimer formation and emission. This is also observed in the EL spectra. The differences in the EL and PL spectrum change upon $\mathrm{Alq}_{3}$ doping are understood in terms of higher sensitivity of the EL to the chain separation. The excimer formation rate in presence of charged molecules in drift regime is strictly interchain distance dependent, since the charge transport is mainly across the polymer chains. Instead, the diffusion length of the neutral excitations (as in PL) and the successive excimer formation turn out to be less influenced by the chain separation, since the transport is mainly along the polymer chains.

The contribution of intrachain and interchain excitations to the blend emission for varying $\mathrm{Alq}_{3}$ percentages $(0 \%, 9 \%$, and $60 \%$ ) is investigated by carrying out luminescence measurements with various time windows (i.e. delay intervals) after the excitation (Fig. 4). The delay intervals run from the excitation pulse ( 0 ps delay) to 960 ps ("FAST" range), from $\sim 2.5$ delay to 17 ns ("MEDIUM" range), from $\sim 62$ delay to $136 \mathrm{~ns}$ ("SLOW" range) and from the excitation pulse to 136 ns delay (quasi-continuous wave, "quasi-CW" range, i.e., the most extended measurable time interval at the used resolution). In the FAST range, the fluorescence from the three samples exhibit similar characteristics (first column). The signal is dominated by MEH-PPV intrachain emission with lifetime of few hundreds of picoseconds, with the $(0 \rightarrow 0)$ and $(0 \rightarrow 1)$ fluorescence transitions visible at 570 and $610 \mathrm{~nm}$, respectively. The emission from pure $\mathrm{MEH}-$ PPV (FAST range) is less structured and exhibits overlapped interchain and intrachain emission components. Already within the first nanosecond after the excitation pulse the more compact structure proper to the $0 \%$ film allows a strong interchain interaction which results in a stronger excitation transfer and a favored MEH-PPV excimer formation. The less structured fluorescence spectrum of the $0 \%$ sample reflects the presence of lower energy overlapped components. By properly choosing the time ranges of fluorescence signal 


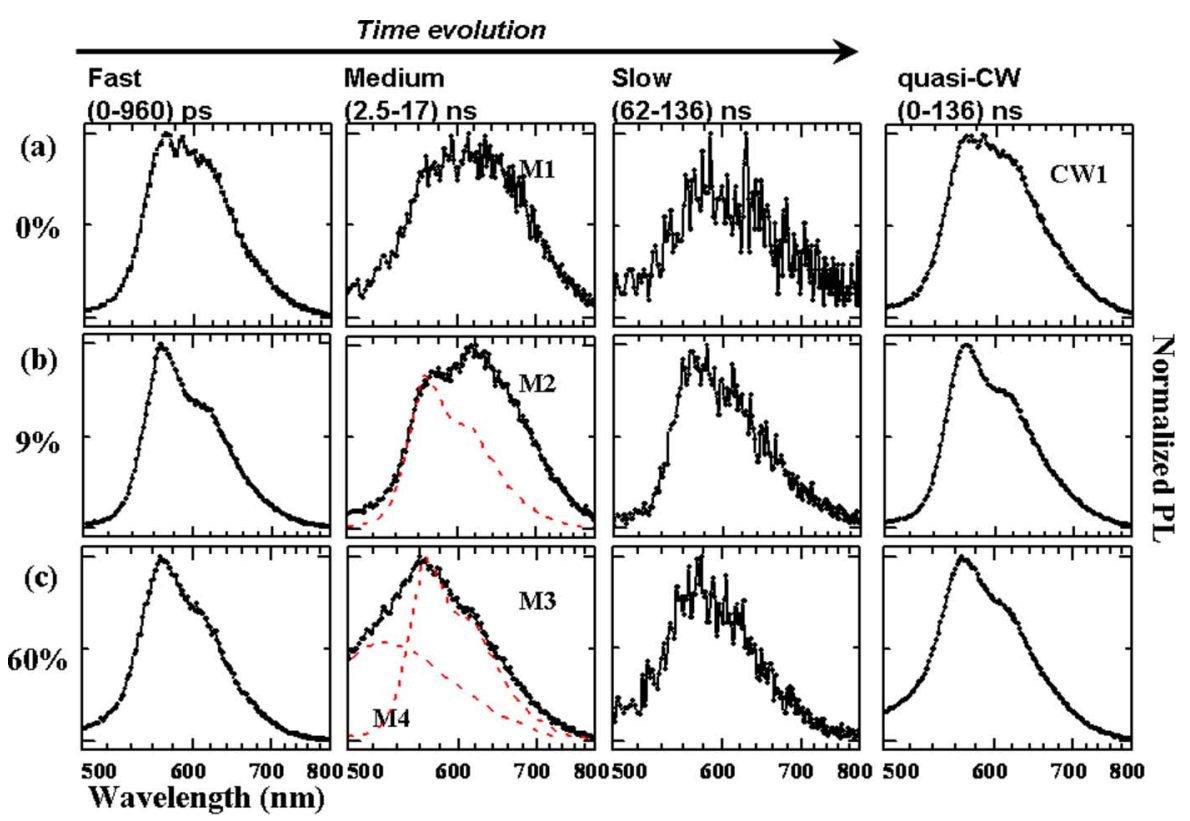

FIG. 4. (Color online) Time resolved PL of MEH-PPV alone (a), MEH-PPV : $\mathrm{Alq}_{3}=9 \% \quad$ (b), and MEH-PPV : $\mathrm{Alq}_{3}=60 \%$ (c). The curves correspond to PL spectrum collected in various time intervals after the excitation pulse. All the spectra are normalized to their maxima. The notations $\mathrm{M} 1$ to $\mathrm{M} 3$ refer to the excimer emission component in the spectra, while M4 refers to the $\mathrm{Alq}_{3}$ emission contribution. collection, the nature of the emitting species in the blend becomes apparent: in the case of pure MEH-PPV [Fig. 4(a)], the intrachain fluorescence evolves rapidly in an unstructured fluorescence centered at 640-660 nm (Fig. 4, M1), assigned to MEH-PPV excimers. ${ }^{11}$ In the case of the $9 \%$ and $60 \%$ blend the excimer component is reduced (M2) and tends to disappear (M3) when the MEH-PPV excimer formation is nearly completely hindered by the presence of large amount of $\mathrm{Alq}_{3}(60 \%)$. In this last case, $\mathrm{Alq}_{3}$ emits independently from MEH-PPV and its fluorescence contribution (centered at $530 \mathrm{~nm}$ ) is seen as overlapped (M4) to that of MEH-PPV. The overlapping spectra of $\mathrm{Alq}_{3}$ and $\mathrm{MEH}-\mathrm{PPV}$ intrachain fluorescence are traced by dashed lines as a guide to the eye. The observed $\mathrm{Alq}_{3}$ fluorescence lifetime amounts to $\sim 2 \mathrm{~ns}$ (at $510 \mathrm{~nm})$, i.e., shorter than what is usually measured $(\tau$ $\sim 10-12 \mathrm{~ns}$ ) at room temperature. An excitation transfer from $\mathrm{Alq}_{3}$ to MEH-PPV takes place with an estimated rate of $k_{\mathrm{t}} \sim 5 * 10^{+10} \mathrm{~s}^{-1}$ at $60 \%$ content and, since no $\mathrm{Alq}_{3}$ fluorescence is detectable, at still higher rate for lower doping levels. The SLOW fluorescence signal (third column), turns out to have, again, predominantly an intrachain MEH-PPV character. This possibly reflects a thermally activated dissociation of MEH-PPV excimers, followed by a "delayed" intrachain $\mathrm{MEH}-\mathrm{PPV}$ fluorescence $\left(\mathrm{MM}^{*} \rightarrow \mathrm{M}+\mathrm{M}^{*} \rightarrow \mathrm{M}+\mathrm{M}+\mathrm{h} \nu\right)$. The apparent fluorescence lifetime turns out to be tens of nanoseconds, i.e., nearly three orders of magnitude longer than the "prompt" fluorescence lifetime of intrachain MEH-PPV species as observed within the first time interval. Noticeably, the different time evolution of the fluorescence of the three different blends has, in the integrated spectra (Fig. 4), less evidence. What is observed in the quasi-CW spectra reflects nevertheless the change in steady state EL (Fig. 2) and PL spectra (Fig. 3) upon increasing $\mathrm{Alq}_{3}$ content. As a result, we can infer that the modulation observed in the steady state emission spectra of the blends mainly originates from the different contributions to the fluorescence deriving from excited interchain species of MEH-PPV, i.e., excimers states.

Progressively passing from low concentrations to more than $50 \% \mathrm{Alq}_{3}$ in the blend, it is possible to tune the formation of MEH-PPV excimers (by $\mathrm{Alq}_{3}$ molecules interposition between polymer segments) and, in the limit of $\mathrm{Alq}_{3}$ high concentration, to hinder it totally. Whereas the steady state spectrum does not show any evident contribution of $\mathrm{Alq}_{3}$ fluorescence, by using detection techniques with time resolution and by properly choosing the delay ranges it is possible to evidence such a contribution (M4).

In this work, a blend consisting of MEH-PPV as hole conductor and emitting species and $\mathrm{Alq}_{3}$ as electron conductor has been investigated by EL, steady state and timeresolved fluorescence spectroscopy, upon variation of $\mathrm{Alq}_{3}$ content. As the $\mathrm{Alq}_{3}$ content in the blend increases, the interchain contribution to EL and PL is reduced with respect to the intrachain one, since the presence of $\mathrm{Alq}_{3}$ molecules augments the distance between the MEH-PPV chains, progressively hindering the excimer formation. TRPL evidences the dynamics of excitation transfer from intrachain to interchain species in MEH-PPV and from $\mathrm{Alq}_{3}$ to MEH-PPV when the $\mathrm{Alq}_{3}$ concentration is increased.

This work was supported by the Spanish Ministry of Education and Science through Grant No. TEC2006-10665/ MEC, the Consolider-Ingenio Grant No. CSD2007-00007 and the Ramon y Cajal program for S.C. E.L. acknowledges Emilia-Romagna Region PRRIITT NANOFABER.

${ }^{1}$ P.-I. Shih, Y.-H. Tseng, F.-I. Wu, A. K. Dixit, and C.-F. Shu, Adv. Funct. Mater. 16, 1582 (2006).

${ }^{2}$ T. Kambayashi, H. Wada, M. Goto, T. Mori, B. Park, H. Takezoe, and K. Ishikawa, Org. Electron. 7, 440 (2006).

${ }^{3}$ S. Quan, F. Teng, Z. Xu, L. Qian, and Y. Wang, Solid-State Electron. 50, 1506 (2006)

${ }^{4}$ J. H. Ahn, C. Wang, C. Pearson, M. R. Bryce, and M. C. Petty, Appl. Phys. Lett. 85, 1283 (2004).

${ }^{5}$ K.-M. Yeh and Y. Chen, Org. Electron. 9, 45 (2008).

${ }^{6}$ Y. Zhang, J. Peng, Y. Mo, and Y. Cao, Appl. Phys. Lett. 85, 5170 (2004). ${ }^{7}$ G. He, Y. Li, J. Liu, and Y. Yang, Appl. Phys. Lett. 80, 4247 (2002).

${ }^{8}$ M. Yan, L. J. Rothberg, F. Papadimitrakopoulos, M. E. Galvin, and T. M. Miller, Phys. Rev. Lett. 73, 744 (1994).

${ }^{9}$ M. Pope and C. E. Swenberg, Electronic Processes in Organic Crystals (Oxford University Press, New York, 1982).

${ }^{10}$ T.-Q. Nguyen, V. Doan, and B. J. Schwartz, J. Chem. Phys. 110, 4068 (1999).

${ }^{11}$ Y. Shi, J. Liu, and Y. Yang, J. Appl. Phys. 87, 4254 (2000).

${ }^{12}$ R. Jakubiak, C. J. Collison, W. C. Wan, L. J. Rothberg, and B. R. Hsieh, J. Phys. Chem. A 103, 2394 (1999). 